\title{
HIV Testing During Pregnancy: Use of Secondary Data to Estimate 2006 Test Coverage and Prevalence in Brazil
}

\begin{abstract}
Célia Landmann Szwarcwald ${ }^{1}$, Aristides Barbosa Júnior², Paulo Roberto Borges de Souza-Júnior ${ }^{3}$, Kátia Regina Valente de Lemos $^{4}$, Paulo Germano de Frias ${ }^{5}$, Karin Regina Luhm ${ }^{6}$, Marcia Moreira Holcman ${ }^{7}$ and Maria Angela Pires Esteves ${ }^{8}$ ${ }^{1}$ Health Information Laboratory, Health Science and Technology Information and Communication, Oswaldo Cruz Foundation; ${ }^{2}$ Monitoring and Evaluation Unit, Brazilian Program of STD and AIDS; ${ }^{3}$ Health Information Laboratory, Health Science and Technology Information and Communication, Oswaldo Cruz Foundation, Brazil; ${ }^{4}$ Evandro Chagas Research Institute, Oswaldo Cruz Foundation, Brazil/State Health Department of Rio de Janeiro; Rio de Janeiro, RJ; ${ }^{5}$ Maternal-Child Institute of Pernambuco, IMIP; Recife, PE; ${ }^{6} \mathrm{Health}$ Secretary of Curitiba, SMS-Curitiba, Federal University of Paraná; Curitiba, PR; ${ }^{7}$ State Health Secretary of São Paulo; São Paulo, SP; ${ }^{8}$ Health Information Laboratory, Health Science and Technology Information and Communication, Oswaldo Cruz Foundation; Rio de Janeiro, RJ, Brazil
\end{abstract}

\begin{abstract}
This paper describes a methodological proposal based on secondary data and the main results of the HIV-Sentinel Study among childbearing women, carried out in Brazil during 2006. A probabilistic sample of childbearing women was selected in two stages. In the first stage, 150 health establishments were selected, stratified by municipality size $(<50,000 ; 50,000-399,999 ; 400,000+)$. In the second stage, $100-120$ women were selected systematically. Data collection was based on HIV-test results registered in pre-natal cards and in hospital records. The analysis focused on coverage of HIV-testing during pregnancy and HIV prevalence rate. Logistic regression models were used to test inequalities in HIV-testing coverage during pregnancy by macro-region of residence, municipality size, race, educational level and age group. The study included 16,158 women. Results were consistent with previous studies based on primary data collection. Among the women receiving pre-natal care with HIV-test results registered in their pre-natal cards, HIV prevalence was $0.41 \%$. Coverage of HIV-testing during pregnancy was $62.3 \%$ in the country as a whole, but ranged from $\mathbf{4 0 . 6 \%}$ in the Northeast to $85.8 \%$ in the South. Significant differences according to race, educational level and municipality size were also found. The proposed methodology is low-cost, easy to apply, and permits identification of problems in routine service provision, in addition to monitoring compliance with Ministry of Health recommendations for pre-natal care.
\end{abstract}

Key-Words: HIV testing, pregnancy, pre-natal care, secondary data, Brazil.

Epidemiologic studies that demonstrate different aspects related to geographic distribution of HIV infection over time provide crucial information for HIV prevention and AIDS management programs. While epidemiologic surveillance of AIDS reflects a situation several years after infection took place, due to the long latency period prior to developing the disease, HIV monitoring shows a more recent picture [1].

Diverse methods have been adopted to estimate the prevalence of HIV infection. The first method proposed, called "back calculation," strives to reconstruct the pattern of HIV infection in the past and predict the number of future AIDS cases [2]. This technique of retrospective estimation depends on the distribution of the incubation period and the AIDS incidence curve over time. Nonetheless, new information about the incubation period, which has gone through important changes as new therapeutic schemes are introduced, can significantly modify estimates of HIV prevalence [3,4].

An alternative method for estimating HIV prevalence was developed by UNAIDS, the Joint United Nations Programme on HIV and AIDS, to compare HIV prevalence among member nations [5,6]. The method is considered direct, since it consists of summing the number of infected cases amongst groups Received on 8 December 2007; revised 4 April 2008.

Address for correspondence: Dr. Célia Landmann Szwarcwald. Health Information Laboratory, Health Science and Technology Information and Communication, Oswaldo Cruz Foundation. Av. Brasil, 4365. Rio de Janeiro, Brazil. Zip code: 21040-360. E-mail: celials@cict.fiocruz.br.

The Brazilian Journal of Infectious Diseases 2008;12(3):167-172. (C) 2008 by The Brazilian Journal of Infectious Diseases and Contexto Publishing. All rights reserved. considered at-risk for infection, with these groups defined by each country. The procedure was improved to take into account variations in size of the at-risk groups and their estimated HIV prevalence rates [7].

In order to monitor the dissemination of HIV infection, an HIV surveillance project was proposed by the former Global Program on AIDS of the World Health Organization and is currently continued by UNAIDS. This project consists in systematically collecting data to establish trends in HIV infection over time and geographic area in certain population sub-groups, considered "sentinel populations" [8].

Pregnant women are considered especially important for this type of study, since, as a group, they are generally representative of all women of reproductive age $[9,10]$. Nonetheless, data collection is generally performed through prenatal health services, considered sentinel sites, and nonrandom selection of health establishments can substantially affect results. In India, HIV prevalence found in a populationbased study was much lower than that obtained through a sentinel study of prenatal clinics [11]. On the other hand, incomplete coverage of the sentinel network in African countries, which fail to reach rural and remote areas, can underestimate HIV prevalence in these countries [12].

In Brazil, HIV prevalence rates have been monitored since 1996 via sentinel studies in health establishments where women are admitted for childbirth. A 1998 analysis of the data collection methodology indicated that non-random sampling of the health establishments (which volunteered to participate in the study) incurred serious limitations for estimating HIV prevalence on a national scale [13]. 
Between 2000 and 2006, the study was biannual, using a probabilistic sampling method, different from the previous years, to select the childbearing women and the health establishments to be analyzed [14-16]. Despite changes in methodology, the study maintained the original name: "Sentinel Surveillance Project".

Besides estimating the HIV prevalence rate, the current project also monitors HIV testing coverage during pregnancy. Beginning in 2002, the study included an interview with the women, which included information about their prenatal care, HIV testing during pregnancy, and the women's knowledge, before the birth, of their serologic status [15,16].

The Brazilian Program of STD and AIDS conducts the Sentinel Childbirth Project every two years, selecting a sample, training personnel at the selected health establishments, and carrying out HIV testing with a blood sample collected at birth. Blood sampling has been performed for all the childbearing women selected in the sample. The blood is then taken to a specifically selected laboratory for analysis.

Given the costs associated with carrying out this project on a national scale, as well as the difficulties in collecting and transporting blood samples, and given that in many cases the test had already been performed during pregnancy, a new methodology was proposed in 2006 to estimate HIV prevalence in childbearing women based on secondary data. The new proposal was to systematize information routinely collected in public health maternity hospitals.

This article presents details of this methodological proposal and the main results obtained regarding HIV prevalence and HIV testing coverage during pregnancy.

\section{Material and Methods}

The new methodological proposal of the Sentinel Surveillance Project among childbearing women was based on pooling and systematizing information about HIV and syphilis test results registered on prenatal cards, when available, and on patient hospital charts, for a selected probabilistic sample of childbearing women. The proposal was developed as a joint research project of the Brazilian AIDS Program, with collaboration from the Science and Technology Information Center of the Oswaldo Cruz Foundation (CICT/ FIOCRUZ), financed by UNICEF and CDC-GAP Brazil. The project was approved by the Research Ethics Committee of the Oswaldo Cruz Foundation.

\section{Sampling}

The sample size was determined in order to estimate HIV prevalence in Brazilian childbearing women. Based on 2004 estimates of HIV infection in pregnant women, $0.41 \%$ [16], a sample size of 16,000 pregnant women was calculated to estimate HIV infection with a 95\% confidence interval and a bilateral error of $0.1 \%$.

The sampling process was probabilistic, in two stages. In the first stage, 150 public maternity hospitals were selected. To be eligible for selection, hospitals must have attended more than 500 births in 2005. The population size of the municipality in which the maternity hospital was located (less than 50,000; 50,001-400,000; more than 400,000 inhabitants) was used to stratify the health establishments. In each stratum, 50 health establishments were selected with probability proportional to size, as established by the number of admissions for delivery in 2005. Within each maternity hospital, 100-120 childbearing women were selected upon admission.

\section{Instrument}

A standardized form was developed to collect the following information:

- Data about the childbearing woman: name, live birth registration number, age, schooling, race, municipality of residence;

- Information about prenatal care: data registered on prenatal card, including number of office visits, date of first visit, results and dates of syphilis and HIV tests (when available);

- Information gathered in the hospital records: results and date of syphilis and HIV tests.

Binders containing 30 printed forms along with instructions for filling out the forms were compiled. Each participating hospital completed 4 binders.

\section{Fieldwork}

After probabilistic selection of the maternity hospitals in each stratum, the municipalities in which the health establishments were located were identified and grouped by geographic proximity. Ten experienced public health researchers were selected to coordinate the fieldwork.

These coordinators were responsible for contacting the directors of the selected maternity hospitals, and for recruiting and training personnel in charge of filling out the forms (generally hospital employees).

\section{Data Analysis}

The present study focused on data regarding HIV testing during pregnancy and birth. Results of syphilis testing will be published separately.

Statistical analysis took into account the sampling design, weighting according to geographic distribution of live births, as registered by the Live Births Information System of the Ministry of Health (SINASC). SPSS version 13 was used for statistical analysis.

HIV test coverage during pregnancy was estimated as the proportion of pregnant women that received prenatal care, brought their prenatal cards with them to the hospital when admitted for delivery, and whose test results had been filled out on the card. HIV prevalence was estimated among childbearing women possessing a prenatal card with written HIV test results.

Indicators of HIV test coverage were analyzed by age group (10-19; 20-34; 35+); schooling (incomplete primary school; incomplete secondary school; incomplete post-secondary 
school; complete post-secondary school); race (white, black, Asian, mixed, indigenous); region; population size of municipality of residence (less than 50,000; 50,001-400,000; 400,001+).

To analyze inequalities in HIV testing coverage during pregnancy, simple and multivariate regression models were used, with failure to be tested for HIV during pregnancy as the dependent variable and region of residence, population size of municipality of residence, schooling, race and age group as independent variables.

As recommended by the Ministry of Health, whenever prenatal HIV test results were not available, women should be tested during childbirth. As a result, the proportion of women that were not tested for HIV during either pregnancy or birth was another indicator examined in the study.

\section{Results}

Data were collected from 16,158 childbearing women. Table 1 shows the distribution of the participants according to HIV testing during pregnancy. Note that only $62.3 \%$ of the women had written HIV test results upon admission to the maternity hospitals; $24.6 \%$ had prenatal cards but without HIV test results; 9.5\% received prenatal care but did not bring or did not possess their cards; and 3.5\% had not received prenatal care. HIV test coverage during pregnancy was considered to be $62.3 \%$.

Among women who received prenatal care, brought their prenatal cards and had written test results, HIV prevalence was $0.41 \%$ (Table 2 ).

Figure 1 shows inequalities in HIV testing coverage by macro geographic region and population size of the women's municipality of residence. Comparing coverage of HIV testing during pregnancy by regions, the Northeast had the poorest coverage, $40.6 \%$, followed by the North, with $46.0 \%$. The South had the highest coverage rate, of $85.8 \%$, with the Southeast and Central West showing rates of $73.7 \%$ and $69.9 \%$, respectively. Analyzing according to the population of the municipalities, coverage varied from $52.1-70.8 \%$, with results directly proportional to the size of the municipality, that is, the more populous the municipality, the higher the rate of HIV testing during pregnancy.

Large inequalities in HIV testing coverage during pregnancy were found between women with different levels of schooling: among women who did not complete elementary school, coverage was $48 \%$, while coverage among women who completed secondary school was $70 \%$. This inequality was even worse when evaluating differences in test coverage according to race. There was a steep decline in coverage, with rates ranging from $74 \%$ among white women to $36 \%$ among self-declared indigenous women (Figure 2).

The results of the logistic regression models, with no HIV test as the response variable, are presented in Table 3. The univariate models show significant differences with p values less than 5\% for almost all the variables considered: macro geographic region, population size (excepting the category
50,001-400,000 inhabitants, which did not show significant difference when compared with the category $>400,001$ inhabitants), schooling, race, and age group.

The differences persist in the multivariate model (Table 3), with the exception of the adjusted effect of age group: there was no significant difference between adolescents and women 20-34 years old. Asian and white women also did not show differences. The most significant effects, when comparing region of residence, were found between the North/Northeast regions and the South, followed by the effect of living in less populous municipalities. Among individual characteristics, we draw attention to the unfavorable effects of fewer years of schooling and non-white skin color.

Data presented in Table 4 shows that $18 \%$ of the women in Brazil were not tested for HIV during either pregnancy or childbirth. Regional results show that this indicator varies from $4.8 \%$ in the South, to $37.2 \%$ in the North.

\section{Discussion}

The sentinel group of pregnant women in Brazil was selected to estimate prevalence of HIV infection because, despite limitations suggested by some authors $[17,18]$, it is the population group that best represents infection rates among all women of reproductive age $[9,10]$.

After carrying out three studies based on blood testing in a sample of pregnant women admitted for childbirth, the present study sought to evaluate a methodological proposal to estimate HIV prevalence in childbearing women based on secondary data, using systematized information routinely collected in public maternity hospitals. HIV results collected using this methodology were very similar to Sentinel Study results from 2004; the estimated HIV prevalence of $0.41 \%$ was identical to that calculated in 2004 [16].

It is important to observe, however, that the HIV prevalence was estimated using information written on prenatal cards and not based on childbearing hospitalization records, since the maternity hospitals were not yet implementing the new HIV testing protocol (through 3 tests in case of discordance), which is currently being implanted. Therefore, the use of a single rapid test during childbirth could lead to false-positive results and biased estimates of HIV prevalence.

By using only results filled-out on prenatal cards, this study presents limitations in estimating HIV prevalence. Women who did not receive prenatal care or who were not tested for HIV during pregnancy were not included in the estimation process. Also, the tests were not standardized. On the other hand, this result is the one being considered by the health services to orient prophylactic treatment for infected women.

Regarding HIV test coverage during pregnancy, in 2004, based on the concept of effective coverage [19], HIV test coverage during pregnancy was calculated as the proportion of pregnant women who received at least one prenatal appointment, for whom an HIV test was ordered during the pregnancy, and who received the test result prior to birth [16]. 
Table 1. Distribution of women according to prenatal care and HIV testing during pregnancy. Brazil, 2006.

\begin{tabular}{lrr}
\hline Situation & N & \% \\
\hline Did not receive prenatal care & 573 & 3.5 \\
Received prenatal care but did not bring card & 1,394 & 8.6 \\
Received prenatal care but did not possess card & 146 & 0.9 \\
Had prenatal card but did not have written HIV test results & 3,981 & 24.6 \\
Had prenatal card and written HIV test results & 10,064 & 62.3 \\
Total & 16,158 & 100.0 \\
\hline
\end{tabular}

Table 2. Written HIV test results amongst women who had results marked on prenatal card. Brazil, 2006.

\begin{tabular}{lrrc}
\hline Result & $\mathbf{N}$ & \% & CI (95\%) \\
\hline Negative & 10,022 & 99.59 & $99.361-99.733$ \\
Positive & 42 & 0.41 & $0.267-0.639$ \\
Total & 10,064 & 100.00 & - \\
\hline
\end{tabular}

Table 3. Results of simple and multivariate logistic regression models with failure to be tested for HIV during pregnancy as the dependent variable.

\begin{tabular}{|c|c|c|c|c|}
\hline \multirow[t]{2}{*}{ Variable/Categories } & \multicolumn{2}{|c|}{ Univariate analysis } & \multicolumn{2}{|c|}{ Multivariate analysis } \\
\hline & $\overline{\text { OR }}$ & IC $95 \%$ & $\overline{\text { OR }}$ & IC 95\% \\
\hline \multicolumn{5}{|l|}{ Region } \\
\hline North & $7.09 *$ & 3.72-13.52 & $6.23^{*}$ & 3.34-11.62 \\
\hline Northeast & $8.80 *$ & $5.48-14.13$ & $7.41 *$ & $4.54-12.10$ \\
\hline Southeast & $2.15^{*}$ & $1.30-3.57$ & $2.25^{*}$ & $1.34-3.80$ \\
\hline South & 1.00 & - & 1.00 & - \\
\hline Centre-West & $2.58^{*}$ & $1.39-4.81$ & $2.54^{*}$ & $1.30-4.98$ \\
\hline \multicolumn{5}{|l|}{ Population size } \\
\hline$<50,000$ & $2.23 *$ & $1.48-3.36$ & $1.79 *$ & $1.23-2.60$ \\
\hline $50,001-400,000$ & 1.30 & 0.86-1.96 & 1.34 & $0.95-1.88$ \\
\hline $400,001+$ & 1.00 & - & 1.00 & - \\
\hline \multicolumn{5}{|l|}{ Age group } \\
\hline $10-19$ & $1.15^{*}$ & $1.06-1.26$ & 1.06 & $0.98-1.16$ \\
\hline $20-34$ & 1.00 & - & 1.00 & - \\
\hline $35+$ & $0.82 *$ & $0.71-0.95$ & $0.83^{*}$ & $0.72-0.97$ \\
\hline \multicolumn{5}{|l|}{ Schooling } \\
\hline Incomplete primary school & $2.57 *$ & 2.02-3.28 & $1.82 *$ & $1.51-2.20$ \\
\hline Incomplete secondary school & $1.67 *$ & $1.44-1.94$ & $1.47^{*}$ & $1.29-1.67$ \\
\hline Incomplete post-secondary school & $1.21 *$ & $1.02-1.42$ & $1.17 *$ & $1.01-1.35$ \\
\hline Complete post-secondary school & 1.00 & - & 1.00 & - \\
\hline \multicolumn{5}{|l|}{ Race } \\
\hline White & 1.00 & - & 1.00 & - \\
\hline Black & $2.11 *$ & $1.59-2.79$ & $1.55^{*}$ & $1.23-1.94$ \\
\hline Asian & $2.77 *$ & $1.81-4.23$ & 1.33 & $0.94-1.89$ \\
\hline Mixed & $2.36^{*}$ & $1.92-2.90$ & $1.32 *$ & $1.09-1.60$ \\
\hline Indigenous & $5.20 *$ & 2.17-12.42 & $2.44^{*}$ & $1.35-4.42$ \\
\hline
\end{tabular}

* OR significaantly different from 1 at $1 \%$ level of significance.

Table 4. HIV test coverage during prenatal care or childbirth hospitalization by Region. Brazil, 2006.

\begin{tabular}{|c|c|c|c|c|c|c|c|c|}
\hline \multirow[t]{2}{*}{ Region } & \multicolumn{2}{|c|}{ Not tested } & \multicolumn{2}{|c|}{$\begin{array}{c}\text { Test during } \\
\text { prenatal care }\end{array}$} & \multicolumn{2}{|c|}{$\begin{array}{c}\text { Test during childbirth } \\
\text { hospitalization }\end{array}$} & \multicolumn{2}{|c|}{ Total } \\
\hline & $\mathbf{N}$ & $\%$ & $\mathbf{N}$ & $\%$ & $\mathbf{N}$ & $\%$ & $\mathbf{N}$ & $\%$ \\
\hline North & 616 & 37.2 & 761 & 45.9 & 280 & 16.9 & 1,657 & 100.0 \\
\hline Northeast & 1,493 & 30.9 & 1,963 & 40.6 & 1,375 & 28.5 & 4,832 & 100.0 \\
\hline Southeast & 637 & 10.1 & 4,647 & 73.7 & 1,022 & 16.2 & 6,306 & 100.0 \\
\hline South & 102 & 4.8 & 1,842 & 85.8 & 203 & 9.5 & 2,147 & 100.0 \\
\hline Centre-West & 193 & 15.9 & 851 & 70.0 & 172 & 14.1 & 1,216 & 100.0 \\
\hline Total & 3,041 & 18.8 & 10,064 & 62.3 & 3,052 & 18.9 & 16,158 & 100.0 \\
\hline
\end{tabular}


Figure 1. HIV test coverage during prenatal care by Region and population size of municipality of mother's residence. Brazil, 2006.

HIV test coverage by Region. Brazil, 2006

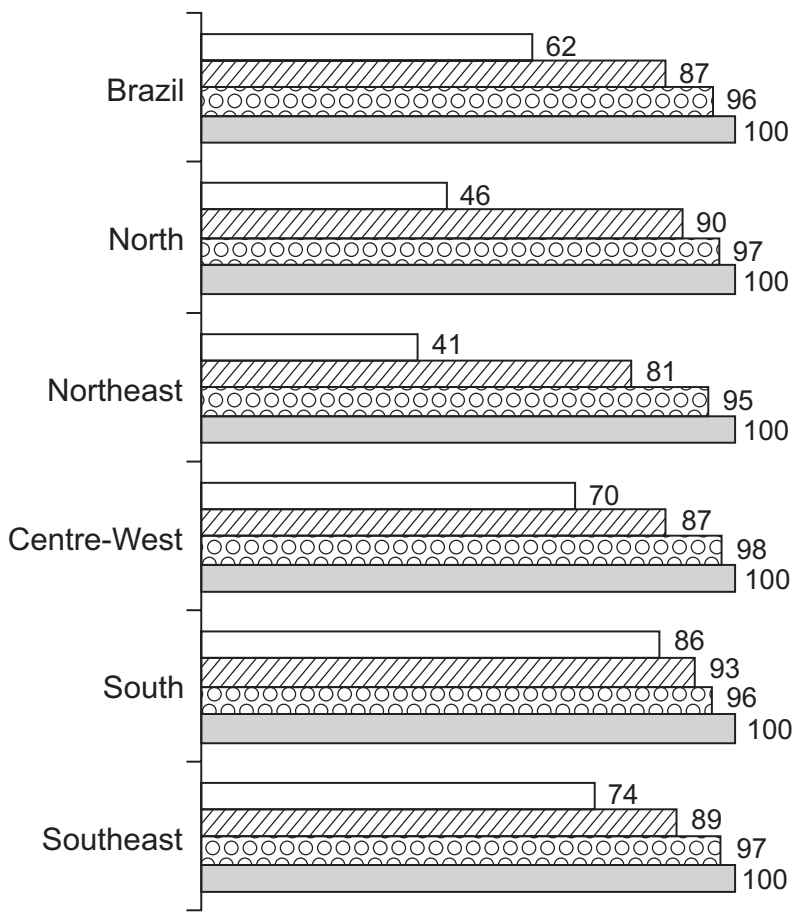

HIV test coverage by municipality population size. Brazil, 2006

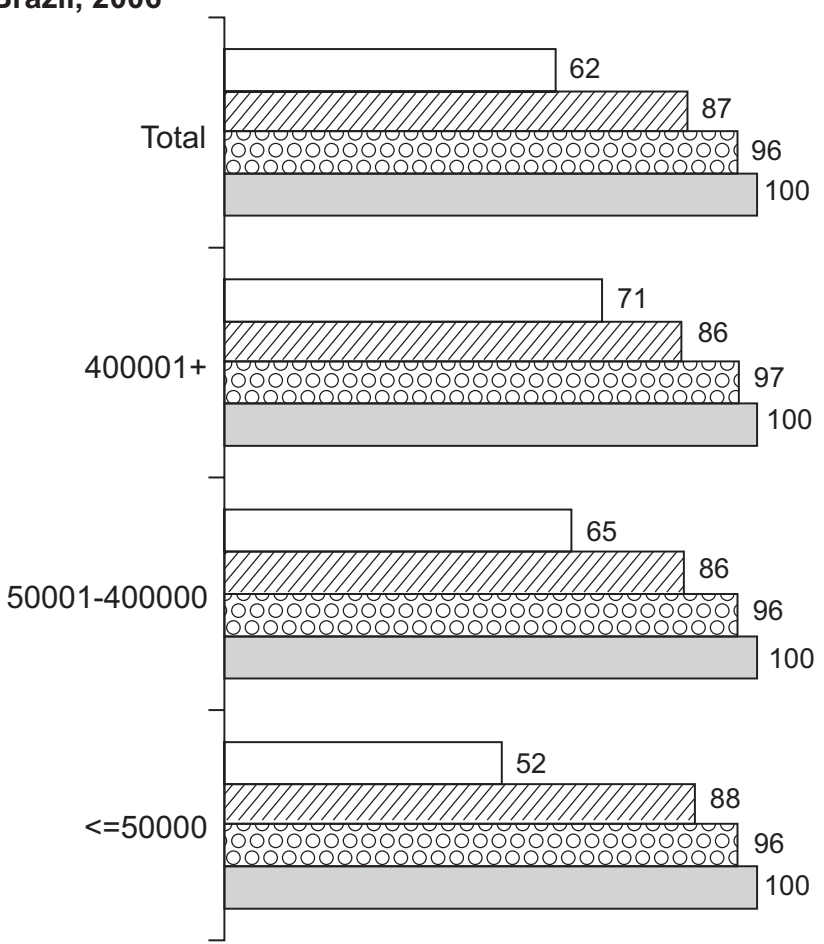

Figure 2. HIV test coverage during prenatal care by mother's schooling and race. Brazil, 2006.

HIV test coverage by schooling. Brazil, 2006

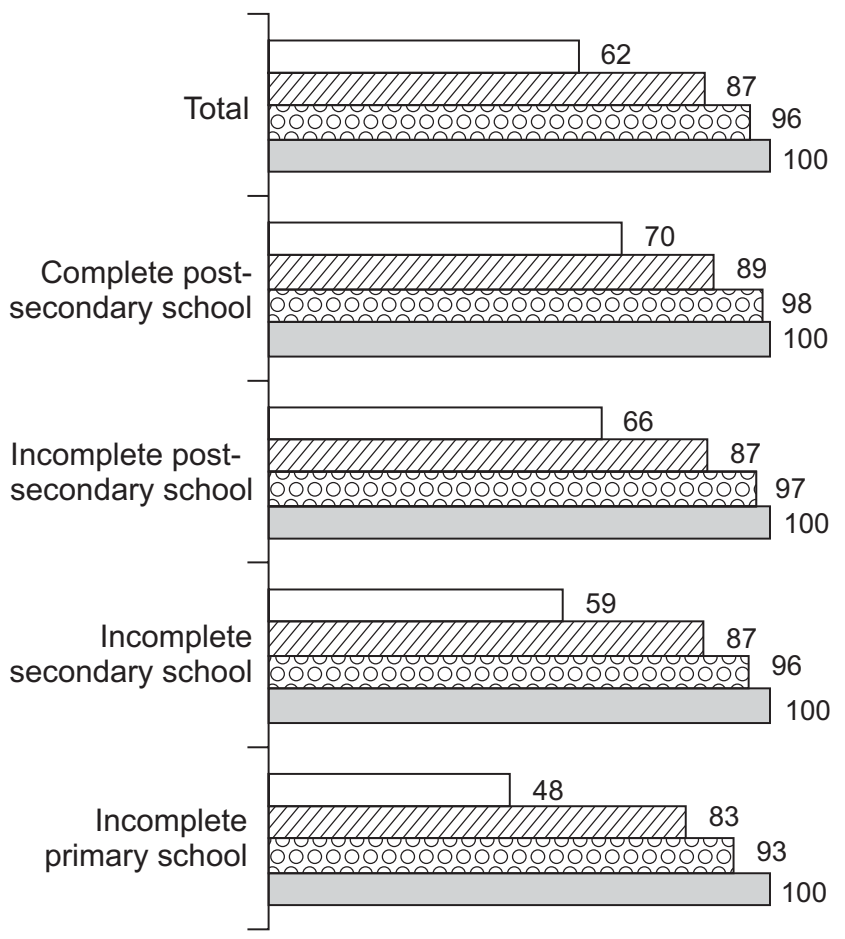

HIV test coverage by race. Brazil, 2006

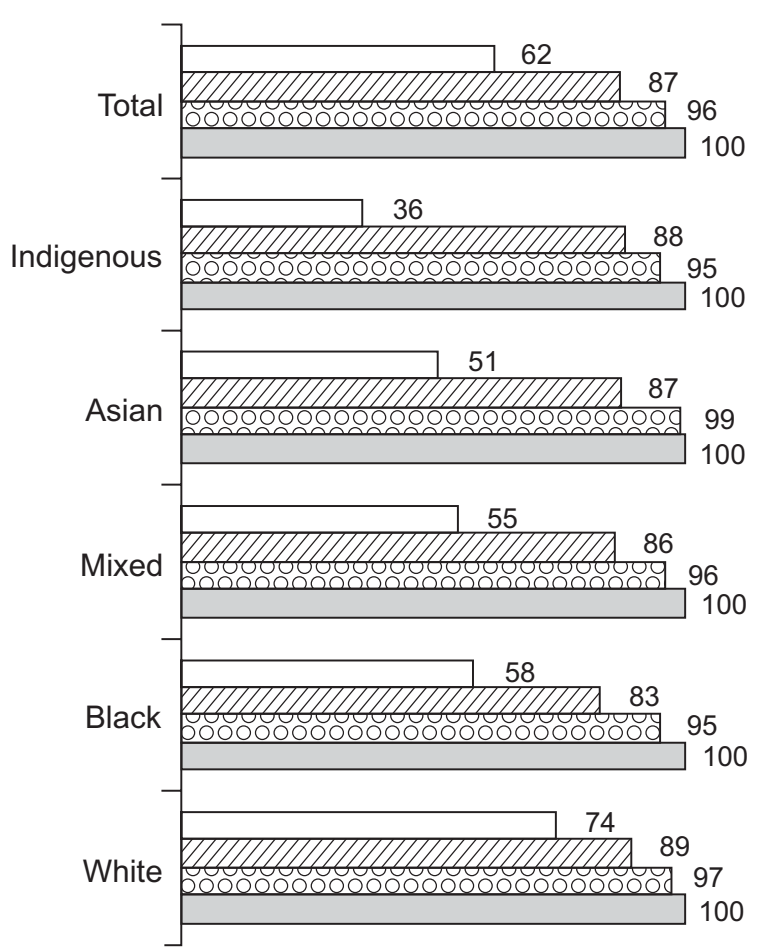

Total $\mathscr{O}$ Prenatal care $\square$ Prenatal card $\square$ HIV test 
The value obtained in 2004 was 63\%. In 2006, HIV test coverage during pregnancy was estimated as the proportion of pregnant women who received at least one prenatal appointment, brought their prenatal card to the maternity hospital and had the test result filled out on the card. The value obtained in 2006 was 62\%. Although different methods were used, the first based on an interview with the childbearing woman and the second based on information registered on the card, the results were very similar.

Results presented here indicate that the proposal for monitoring HIV test coverage and prevalence of HIV during pregnancy using routinely collected data is viable. In addition, this methodological proposal can be carried out at the state and municipal level without the sampling process, in order to monitor compliance with Ministry of Health recommendations.

Inequalities found in previous studies $[15,16]$ relative to HIV test coverage during pregnancy persist. While coverage in the North and Northeast regions did not reach $40 \%$, in the Center-South coverage reached $70 \%$, demonstrating regional inequalities in access to health actions.

While access to prophylactic measures to reduce vertical transmission of HIV is universal in Brazil, there is a social gradient for HIV testing during pregnancy, which discriminates against women with less education and non-whites, due to lack of information and access to resources available through the health system. It is important to emphasize that failure to detect HIV infection during prenatal care represents a lost opportunity for intervention in the infected woman, and limits the chance to reduce the incidence of pediatric cases infected by maternal-child transmission.

In Brazil, the rate of vertical transmission is around 7\%, ranging from $6 \%$ in the South and Central-West to $15 \%$ in the Northern Region, according to results of a Brazilian Pediatric Society study published by the Brazilian AIDS Program (www.aids.gov.br/monitoraids). These rates could reach as little as $1 \%$ if all the Ministry of Health recommendations to avoid vertical transmission of HIV were adopted, including ARV prophylaxis during and after pregnancy [20-22].

In conclusion, AIDS programs at all levels (national, state and municipal) should turn their efforts toward expanding the reach of prenatal testing, with special attention given to certain population groups, in order to make the available resources accessible to socially disfavored women and residents of less developed regions of the country.

\section{References}

1. Mertens T.E., Low-Beer D. HIV and AIDS: where is the epidemic going? Bull World Health Organ 1996;74(2):121-9.

2. Brookmeyer R., Liao J.G. Statistical modelling of the AIDS epidemic for forecasting health care needs. Biometrics 1990;46(4):1151-63.
3. Venkatesan P. A comprehensive back-calculation framework for estimation and prediction of HIV/AIDS in India. J Commun Dis 2006;38(1):40-56.

4. Sweeting M.J., De Angelis D., Aalen O.O. Bayesian back-calculation using a multi-state model with application to HIV. Stat Med 2005;24(24):3991-4007.

5. Walker N., Stanecki K.A., Brown T., et al. Methods and procedures for estimating HIV/AIDS and its impact: the UNAIDS/WHO estimates for the end of 2001. AIDS 2003;17(15):2215-25.

6. Ghys P.D., Brown T., Grassly N.C., et al. The UNAIDS Estimation and Projection Package: a software package to estimate and project national HIV epidemics. Sex Transm Infect 2004;80(Suppl 1):i5-i9.

7. Brown T., Grassly N.C., Garnett G., Stanecki K. Improving projections at the country level: the UNAIDS Estimation and Projection Package 2005. Sex Transm Infect 2006;82(Suppl 3):iii34-iii40.

8. WHO. Carrying out HIV Sentinel Surveillance. World Health Organization 1994; New Delhi India.

9. Colembunders R.L., Heyward W.L. Surveillance of AIDS and HIV infection: opportunities and challenges. Health Policy 1990;15:1-11.

10. Karon J.M., Rosenberg P.S., Mcquillan G., et al. Prevalence of HIV infection in the US, 1984 to 1992. JAMA 1996;276:126-31.

11. Dandona L., Lakshmi V., Kumar G.A., Dandona R. Is the HIV burden in India being overestimated? BMC Public Health 2006;206:308.

12. Yahya-Malima K.I., Olsen B.E., Matee M.I., Fylkesnes K. The silent HIV epidemic among pregnant women within rural Northern Tanzania. BMC Public Health 2006;276:109.

13. Szwarcwald C.L., Castilho E.A. Estimativa do número de pessoas de 15 a 49 anos infectadas pelo HIV, Brasil, 1998. Cadernos de Saúde Pública 2000;16(Sup.1):135-41.

14. Szwarcwald C.L., Carvalho M.F. Estimativa do número de indivíduos de 15 a 49 anos infectados pelo HIV, Brasil, 2000. Bol Epidemiol DST/AIDS 2001;Ano XIV n ${ }^{\circ} 01$.

15. Souza-Jr. P.R.B. de, Szwarcwald C.L., Barbosa-Jr A., et al. Infecção pelo HIV durante a gestação: estudo-Sentinela Parturiente, Brasil, 2002. Revista de Saúde Pública 2004;38(6):764-72.

16. Szwarcwald C.L., Souza-Jr P.R.B. de. Estimativa da prevalência de HIV na população brasileira de 15 a 49 anos, 2004. Bol Epidemiol DST/AIDS 2006; Ano III $n^{\circ} 01$.

17. Boisson E., Nicoll A., Zaba B., Rodrigues L.C. Interpreting HIV seroprevalence data from pregnant women. Journal of Acquired Immune Deficiency Syndromes and Human Retrovirology 1996;13(5):434-9.

18. Glynn J.R., Buvé A., Caraël M., et al. Decreased fertility among HIV-1-infected women attending antenatal clinics in three African cities. JAIDS 2000;25:345-52.

19. Tanahashi T. Health services coverage and its evaluation. Bull World Health Organ 1978;78:1246-55.

20. Mandelbrot L., Le Chenadec J., Berrebi A., et al. Perinatal HIV-1 transmission: interaction between zidovudine prophylaxis and mode of delivery in the French Perinatal Cohort. JAMA 1998;280(1):55-60.

21. Mandelbrot L., Landreau-Mascaro A., Rekacewicz C., et al. Lamivudine-zidovudine combination for prevention of maternalinfant transmission of HIV-1. JAMA 2001;285:2083-93.

22. Ministério da Saúde. Secretaria de Vigilância Epidemiológica. Programa Nacional de DST e AIDS. Recomendações para profilaxia da transmissão vertical do HIV e terapia antiretroviral em gestantes, 2002/2003. Brasília (DF): Ministério da Saúde; 2003. 